\title{
El Método de los Seminarios en el Perú.
}

CUANDO SE ACORDO LA CREACION DEL PRIMER

SEMINARIO

En la sesión de la Facultad de Letras del dia i 7 de enero de 1925 se acordó, do quteca continuacióncopio literalmente:

"E1. señor Subdecano después de agradecer el voto de honnr que se le otorgaba, propuso lo creación de un curso de Seminario anexo a la cátedra de Filosofía de la Educación. IEl cloctor Lberico ofreció su concurso para esta forma de estudios; y el doctor Urteaga expresó la conveniencia de que se abriese un curso de Seminario para la asignatura de Arqueología.

La Facultad acordó la creación del Seminario propuesto por el señor Subdecano".

En sesión de la Facultad, de fecha 55 de febrero, la Junta acordó el nombramiento de Jefe de Trabajos Prácticos de 
Filosofia de la Ẽducación, en el Seminario de Pedagogia. Len sesión de fecha 2 de mayo, se nombró para ese cargo al señor Elias Ponce Rodriguez.

L.a inauguración del Siminario de Pedagogi ia se realizó el 13 de junio de 1925 y de esta actuación dió cucnta "El Comercio", en su edición de 15 de junio, en la siguiente forma:

\section{EN LA FACULTAD DE LETRAS}

\section{Inauguración del Seminario de Pedagogia}

"En la tarde del síbado tuvo lugar en la Facultad de Fi.losofía, Historia y Letras, la inauguración del "Seminario de Pedagogía", establecido por el doctor Luis Miró Quesada catedrático de Filosofía de la Educación en esa Facultad; y destinado a realizar, con alumnos que tengan ya los conocimientos que en ese curso se enscinan, estudios especiales de investigación psico-jeclagógica. "Antes de comenzar stis liabores el grupo de alummos que constituye el "Seminario de Pedagogía" en referencia, dijo el doctor Miró Quesada; que deseaba precisar el origen, el objeto y los propósitos del Seminario que se inauguraba y determinar cl criterio que debia guiar sus estudios e investigaciones".

Se refiere al origen, objeto y propúsitos del Seminario, y más adelante prosigue así, en esta lección inaugural:

"La circunstancia, dice el cloctor Miró Quesada, de haber realizado en años anteriores en la cátedria de "Filosofía de la Educación" trabajos de investigación psico-pedagógica, me hizo pensar en la conveniencia de fundar un "Seminario de Pedagogía", en el cual un pequeño número de alumnos, con interés especial pòr los estudios de esta índole, pu- 
dieran hacer en condiciones adecuadlas, laloor activa y personal. TEl año pasado, en efecto, encargué a los alummos señores E. Ponce Rodríguez, E. Espinoza, G. García y H. Luna, dirigiclos por el primero de los nombrados, la determinación del grado de sugestibilidad de los niños de nuestras escuelas prinarias, aplicando, con tal objeto los "Tests de sugestibiliclad" de Binet. Estc ensayo dió muy interesantes resultados: pero no podia repetirse sino ocasionalmente en una clase que, como la de "Filosotia de la Educación", está destinadia a clar una cultura general pedagógica a los jóvenes universitarios cue por clla pasan. Era, pues, necesario crear un curso de Seminario, con tendencia a la investigación experimental, y al que concurriera un grupo de alumnos con particular vocación pedagógica y preparado para el género de trabajos que debia efectuar.

"Con estas tendencias, agrega el cloctor Luis Miró Quesada, la Facultad de Filosofít, Historia y Letras, aprobó en su sesión de is de cnero del año en curso, la creación de un "Seminario de Pedagogía que debia dirigir el catedrático de "Tilosofía de la Educación"ücsólicitócolarvez del Consejo de Facultades, autorización para nombrar un jefe de trabajos prícticos para el referido Seminario, designando, más tarde, para desempeñar el cargo al señor Elías Ponce Rodríguez. Mientras tanto se ha preparado el aula especial, donde debe apuel funcionar.

"Tales los antecedentes principales del Seminario de Pedagogia que en la Facultad de Letras se inaugura. Fn cuanto al objeto que él persigue de realizar investigaciones experimentales de inclole psico-pedagógica, creo que se satisface bien esta finalidad, tratando este año cl siguiente tema general: "Los tests mentales y su utilidad escolar". Auestra labor, 
pues, se dirigirá a investigar el valor psicológico y pedagógico de los tests y la forma y condiciones en gue pueden ser aplicados a nuestrás escuclas primariats, para medir la inteligencia de nuestros escolares y establecer su cliferencia de capacidad mental. Si esto es posible obtenerlo, habrá llegado el monento cle estudiar la forma en que la enseñanza primaria se dé en nuestras escuclas en condiciones realmente psicoloricas; o sea, de modo, que se adapte a las diversas concliciones de capacidad cle los niños; y pueda, por consiguiente, ser eficazmente aprovechada por todos".

Se extiende ensegtida, sobre el interés que tienen los "tests" desde el punto de vista científico, y prosigue así:

"Con este criterio hemus de proceler a investigar el valor psicológico y pedagógico de los "tests" antes de utilizarlos en la tarea de medir la inteligencia o aptitudes de nuestros escolares; $y$ cuando estemos en condiciones de emprender esta labor, los resultaclos obtenidos serán sometidos a un estut. dio critico para que podamos considerarlos como definitivos. Nuestro principal objeto se dirigira a cleteminar el "grado de inteligencia" "le fos niños de las escuelas públicas. Para ese propósito, creo que no habrá inconveniente en utilizar como fundamentales: el "tests" individual de Terman (la revisión de la escala métrica de Pinet) y los colectivos llamados "national tests", que están ya debiclamente estanclarizaclos y tienen hoy universal aceptación. Puede ser empleado, en ciertos casos, y crmmo auxiliares los "tests" de Sanctis y de Portens.

"En lo que se refiere a los "tests" peclagógicos, objetivos o de instrucción, los más tusados son los de Thornclike, Curtis y Aires, para medir el gradio de habiliclad escolar en aritmética, escritura y lectura; y será interesante cnsayar la 
aplicación de algumo de ellos a los niños de nuestras escuelas. Más dificil ciertamente, es el empleo de los "tests" de aptitudes, que aspiran a servir de guía para la orientación profesional. Es este un problcma qute, no obstante su importancia. no ha sido definitivamente resuelto todavía. Y se compren. de: determinar una aptitud, no significa como dice Claparede hacer un diagnóstico, sino efectuar un pronóstico; o sea vaticinar, previendo el porvenir, para qué va a servir un niño más tarcle. Nos importa, por eso, seguir, prudentemente, el intenso movimiento de investigación que en tal sentido se realiza hoy en listaclos Lniclos y Eutopa.

"Concluyó, el cloctor Miró Quesada manifestando que. al declarar inaugurados los trabajos del "Seminario de Pecladogía", confiaba en que ellos serim fructíferos si un entusiasmo ponderaclo guia la conún labor".

\section{I,A LABOR REALIZADA EN EL SEMUNARIO DE PEDAgOgIa}

Para facilitár qaspabor que realizabans los estudiantes, el catedrático del curso de Filosofía de la Educación, doctor Luis Miró Quesada. cscribió una serie de artículos de divulgación sobre "Los "tests" mentales aplicados a la educación" que fueron publicados scmanalnente en el diario "El Comercio", a partir del 2 de agosto de 1925. Para que el lector pulda clarse cuenta de la importancia de estos artículos, enumerare ínicamente los titulos:

I.- "I Ia P'sicología Experimental y los "Tests" mentales.

II._- "Valor" del "Tests" Mental como Método Psicológico". 

les".

IIJ.-- "La Psicología Pedagógica y los "Tests mentaIV._- "Valor del "Tests" como método pedagógico".

V.-. "La medida de la inteligencia".

VI.-." Los deficitntes intelectuales y los supernormales".

VIL.- "J a Lscala Mlétrica de la inteligencia".

VIII._"El Valor permanente de la Escalit Binet-Simón".

IX.- "La Stanford Revisión" de la Escala Binet-Simón1".

X.- "Métodos de medir lá inteligencia distintas a la escala binet-Simón".

XI._. "Los "Tests Colectivos" y su empleo durante Ia Gran Guerra".

XII._-Los "National Intelligence Tests".

XIII._- "Los "I'ests" Objetivos de Instrucción".

XIV.—.La medida de la 'Enseñanza".

XV.- "La Orientación Profesional".

XVI._-"La Orientación Educativa".

X.VII._ "Conveniencián de Gtilizarlos" en el Perú".

Y mientras tanto se continuaba trabajando en el Seminario de Pedagogía en la investigación y selección de los Tests Mentales que mejor convenían al propósito de obtener una medida adecuada y práctica de la inteligencia de los escolares de Lima. Con este objeto fueron traducidos del inglés los "National Intelligence Tests", las Claves y el Manual de Direcciones para aplicarlos adaptándolos a las condiciones de nuestros escolares. Al respecto, el doctor Miró Quesada, cn un artículo aparecido en "El Comercio" el 22 de noviembre de 1925 , sobre la conveniencia de utilizar los "Tests" mentales en el Perú, dice lo siguiente: 
"Pero haly un vasto y fundamental campo en la nueva peclagogia: el que se refiere a la medida de la inteligencia de los cscolares, en el que los llamadios "tests" mentales son la expresion de un problema ya enteramente resuelto. For meclio de ellos es posible cotnocer, en efecto, lo que un niño puede y debe hacer en relación con la enseñanza y de acuerdo con su capaciuad nativa. Con los "National Intelligence "Tesis", por cjemplo, cs fícil electuar en pocas horas, un exanlen colectivo, que permita una exacta clasificación de los uiños de una escuela, y que suministre un criterio exacto paral separar a los normales de los de superior inteligencia, cle los simples retardados y de los deficientes, con el objeto de establecer clases diferentes para cada uno de estos grupos diversos. 'Es posible, asimisno, descubrir en los estudiantes algunas especiales aptitudes intelectuales que anuncian ya cicrta vocación profesional.

"Con estos propósitos, en el Seminario de Pedagogía hemos traducido y adaptado a nuestras necesidades escolares los "National Intcingenced ests" y los estamos aplicando actualmente en qasescurias tiscales/desdima: contando en estal labor, los mienbros de ese centro, con la cooperación entusiasta de la Inspección del Ramo y de algrinos masestros de la capital. Hasta ahora, y. mientras los datos recogidos puedan ser debidamente clasificados e interpretados, sólo es posible anotar el hecho del interés, casi diríamos el agrado con que los niños ejecutan el trabajo que de ellos se demanda y la facilidad y rapidez con que el examen se realiza. Ha sido posible en efecto, hacer pasar por esta prueba colectiva a más de I jo alumnos en dos turnos consecutivos y en menos de hora y media. Si en aquella oportuniclad la capacidad del salón hubiera permiticlo tomar el 
examen conjunto de: la totalidad de aquellos, el ticmpo cmpleado habría podido reducirse a lá mitad.

"Seguramente, la aplicación de los "tests" val a servir, cntre nosotros, para demostrar la gran heterogeneidad, desde el punto de vista de la inteligencia de los alumnos, existente entre las mismas clases de una iscuela, y entre clases semejantes de diferentes escuclas, y es natural que así seá porque este fenómeno se presenta en toxlos los paises. "lin una clase típica de primer año, dice Teman, el alumno deficiente se aproxima a tna edad mental de $404 \mathrm{r} / 2$ años, $y$ $y$ el superimmente inteligente, a la de 8 a $81 / 2$ años. Si tomamos en conjunto, uni docena de clases de prinuer año, la escala es ordinariamente de $303 \frac{1}{2}$ años a ro o $10 \% \frac{1}{2}$. Singularmente, en una docena de clases de tercer año, podrát establecerse una escala de 7 años de edad mental a I.3 años; en clases de quinto año, de 8 años de edad mental a i 8 años, y en clase de octavo año, de 10 años hasta el quinto más elevaclo que cualquiej icscala de la inteligencià puede medir"" En seneral, se calculac que no pasa de, 411 65 por ciento el número de los alummos que están on la clase que realmente corresponde a su capacidad mental; y qule el resto de: ellos se halla uno o más grados más adelantado o atrasado de lo que deberian estar de acuerdo con su inteligencia. Dicons, examinando en Estados Uniclos, clases del mismo grado, en diversas escuelas, encontró, asimismo, que la edad mental merlia cle ellas difería en dos o más años.

"Y esta heterogencidad, en lo que se reficre a las diferencias mentales entre los alumnos de un mismo año de estudio se agrava en el Perú por la clivergencia de edad cronológica existente entre los escolares que asisten a la misma clase. Investigaciones realizadas en el Seminario de Peda- 
gogia, con 2,2,39 niños pertenecientes a cinco distintas escuelas fiscales de Lima, demostraron que, mientras el pro. medio de edad en el $z^{\text {er }}$ año de primaria era de cloce años, había en una cscueda tres niños de 9 y 5 de i 5 años; en otra. 2 de 10 y 5 de 16 ; en otra, una de 8 y 3 de 16 años, etc. Pocos sistcmlis escolares habrá sin duda más necesitaclos qua cl nucstro del atuxilio de los "tests" mentales, como medio de reagrupar a los allumnos en clases homogéneas, de actier(b) con su habilidad nativa, $y$, por consiguiente, de su real capacielard para el estudio".

De acuerdo con lo expresado por el cloctor Miró Quesatla se habína mandado imprimir los "Tests" traducidos y adaptados por el Seminario de Pedagogía, para ser aplicados en las Escuelas de Lima, y que decín lo siguiente, en su caríttula:

"FACUITAD DE LETRAS DE LA UNTVERSTDAD DF T.IMA. - Scminario de Pedagogia". Y tiene una 1lamada que dice: "Las escalas A. y B. de los National Intelligence Tests" hain sido verticlas al castellano, procurando conservar la mayor fidelidad con el original en inglés, salvo las exigencias de adaptación a nuestro país. Este trabajo se ha hecho, en el "Seminario de Pedagogía" de la Facultad re Filosofía, IIsstoria y Letras de la Universiclad de San Marcos. por los profesores doctor Luis Miró Quesada que dirige el Seminario, y señores doctor Luis Borouncle, Elías Ponce Rodríguez, José García Rodríguez y Carlos Velázque\%, que han prestado su valiosa cooperación; habiendo contribuíclo, eficazmente, a la cuicladosa revisión de la labor realizada, los scĩores Enrique Espinoza, Gerardo Aliaga, Gerisino Garcia, Abclón Max Pajuelo, Agustin Luna, Ricarclo leijó, Alberto Arca Parró, Víctor Sosa y señorita Victoria Infante". 
La segunda parte de la labor del Seminatio consistió, pues, en la aplicación de estos "tests" a tres mil escolares de Lima.

Lnego se llevaron estos datos al Seninatio, para la investigación del grado de inteligencia de los niños peruanos.

Se hicicron investigacinnes detenicias de los tres mil catsos, aplicando las reglas establecidas, patra sacar los cuccientes intelectuales cle los escolares.

: Se sacó luego la medida del grado de inteligencia de los niños en los distintos años.

Se publicaron después los ctiadros gráficos, los índices $y$ las conclusiones rleducirlas.

El resultado obtenirlo con la aplicación de los "Tests" a tres mil escolares de T.jma, estí puntualizaclo en el artícnlo que publicó el doctor tuis Mirí Quesacla en "El Comercio" el 2.3 de marzo de In26. y del cual reproduzco los siguientes nítrafos:

"Al finalizar, en noxiembre del año pasarlo, la serje de artículos que escribiéramos, com el título de: "Tos tests mentales aplicaclos a la edtaction". manifestanios que en el "Se-

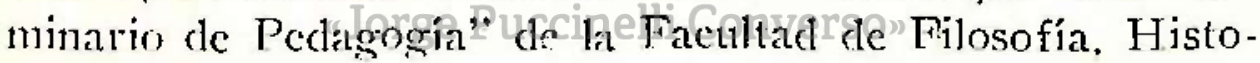
ria y Letras de la Universirlad de San Marcos se habia traduciclo y adaptado a nuestras necesidades escolares los "National Intelligence Tests": y que se estaba ya haciendo con cllos investigaciones en las escuelas de Lima. En los meses trascurriclos, de entonces a la fecha, se ha logrado obtener, cmpleando tal método, algunos resultados respecto a la medida de la inteligencia de nucstros estudiantes, tue cremos ofrece interés darlos a conocer, porcuue se basan en ratos reales y precisos, susceptibles de ser ampliados y aún rectificados más tarcle, pero que constituyen ya funclamento sólicto para derivar de ellos valiosas sugerencias". 
"Fn el Seminario de Pedagogía", y con el nombre de "Tests Colcctivos de Inteligencia" fueron - como en otra oportunidad se hat dicho- verdidos al castellano las escalas A y 1,3 de los "National Intelligence l'ests", procturando conservar la mayor fidelidad con el original en inglés, salvo las exigencias de aldeptación a nuestro medio; $y$ la más exacta similitud, también, en su inpresión. Estando ellos cestinados, en Listados Unidos, al examen de los niños del $33^{\circ}$ al $33^{\circ}$ srato de enseñanza, o sea, aquellos que no han llegatdo a la instruccion secundaria, lat investigacion se ha efectuado entre nosotros con los altumus del tercero al quinto año, inclusive, de primaria. liueron examinaclos, con tal propósito los signtientes niños: 218 , en cl Centro Escolar Pardo; 78 , en la liscuela $4.306 ; 118$ en el Centro Escolar 432 (de mujeres) : etc. etc. (Sigue la relición de escuclas).

"Se ha contadlo en estal labor con el decidido interés hatcial la investigacion que se realizalja, demostrada en forma de amplials facilidacles presiadas a cllat, por el inspector de instruccion, ductur diatvinte ef inspector deleolegio de San Vicente, doctor Oraecliea; effirector del Colegrio de lit Re. coleta, doctor Dintillac; el director del Colegio Anglo Peruano doctor Matc-Lay; el director de lat Escucla Normal (le Varones, doctor bouroncle; $y$ lits directuras y clirectores de Centros Escolares de mujeres y varones, señoritas Aratos y Solari, $y$ señores Filomeno. Tinorain y Espinozil".

"Con el objeto de hacer más comprensible la forma en que hat siclo realizada la investigación pedagógiea de que se trata, vantos a dividir este artículo, en las partes yue at continuación se cxpresan $y^{\prime}$ que trataremos sucintamente: 1 ). El examen en lats escuelas; 2). Calificación de los "tests"; 3). Eil nivel mental de los alumnos; 4). Normas de edad 
mental en relacjón con los alumnos cle los colesios particula res y de las escuelas fiscales; 5 ). Normats de eclad y de gratdo para las clases de $3 .^{n}, 4 \cdot^{n}$ y $5^{\circ}$ años de primaria; 6). Plan de clasificación de los alummos en las clases, de acueroro con su distinta capacidad mental; $y>$ ). Observaciones genera. les". (Desarrolla con extensión toclos estos puntos, qute son el resultado de la investigación realizadda, publicando al respecto varios gráficos).

Es, pues, de está maneral, que se ha llevado a feliz término una investigación con un grupo de estucliantes, quenes en la actualiclad son competentes profesionales. Esta labor científica realizada en la Facultad de Letras y Peclagogia, el año 1925, es tipjicamente de Seminario, a parte de su fundamental utiliclad.

Todo Seminario universitario, hablando en senticto gre. neral, consta de dos partes: el Seminario-Administración y el Seminario-Método.

El Seminario-Administración, es la oficina, dotadí de salones propios, bibljoteca especializald, personal rentado, etc, etc. EI Seminalio-Método, es la marcha por la senda de la investigación.

Lo importante, para que una Universidad pueda llamarse moderna. es rue tenga el Seminario-Mélodo, que puede funcionar aún sin local ni biblioteca, utilizando en estos casos los salones de clase y los libros de la Biblioteca central.

Puede funcionar, también, el Seminario-Adninistración solamente, como sucede en no pocas universidades. En este caso sụ utilidad es casi nula pues sólo sirve para mantener un personal burocratizado.

Lo deseable scría, que ambos seminarios se complemen- 
ten, de tal suerte que las oficinas se esfuercen por prestar todas las facilidades del caso al Seminario-Método.

El primer Seminario que se estableció en la Facultad de Letras, no fué solamente un Seminario-Método, sino que contó con local propio, que estuvo ubicado entre lo que es hoy la Secretaria de la Facultad y el aula del primer año. Contó también con un Jefe de Prícticas rentado.

E) Seminario de Pedagogía de la Facultad de Letras fué crealdo el año 1925, a iniciativa de su Decano el doc1or Luivis Miró Quesada, o sea, hace más de I8 años. Por consiguiente, es el primer Seminario que se organizó en la Universidlad Nacional Mayor de San Marcos, y por ende en el Perín.

Delfín A. I-uneÑa 